\title{
Cellular ubiquitin pool dynamics and homeostasis
}

\author{
Chul-Woo Park E Kwon-Yul Ryu* \\ Department of Life Science, University of Seoul, Seoul 130-743, Korea
}

\begin{abstract}
Ubiquitin (Ub) is a versatile signaling molecule that plays important roles in a variety of cellular processes. Cellular Ub pools, which are composed of free Ub and Ub conjugates, are in dynamic equilibrium inside cells. In particular, increasing evidence suggests that $\mathrm{Ub}$ homeostasis, or the maintenance of free Ub above certain threshold levels, is important for cellular function and survival under normal or stress conditions. Accurate determination of various Ub species, including levels of free $\mathrm{Ub}$ and specific $\mathrm{Ub}$ chain linkages, have become possible in biological specimens as a result of the introduction of the proteomic approach using mass spectrometry. This technology has facilitated research on dynamic properties of cellular Ub pools and has provided tools for in-depth investigation of Ub homeostasis. In this review, we have also discussed the consequences of the disruption of $\mathrm{Ub}$ pool dynamics and homeostasis via deletion of polyubiquitin genes or mutations of deubiquitinating enzymes. The common consequence was a reduced availability of free $U b$ and $a$ significant impact on the function and viability of cells. These observations further indicate that the levels of free $\mathrm{Ub}$ are important determinants for cellular protection. [BMB Reports 2014; 47(9): 475-482]
\end{abstract}

\section{INTRODUCTION}

Ubiquitin ( $\mathrm{Ub})$ is a highly conserved eukaryotic protein composed of 76 amino acids that induces post-translational modification of target substrates by forming isopeptide linkage via actions of Ub-activating enzymes (E1), Ub-conjugating enzymes (E2), and Ub ligases (E3) (1). In general, Ub conjugation results in either monoubiquitination or polyubiquitination of target substrates. Monoubiquitination has been well known to regulate receptor endocytosis and histone modification (2), and polyubiquitination plays diverse functions that are dependent on the type of Ub chain linkages, including degrada-

${ }^{*}$ Corresponding author. Tel: +82-2-6490-2674; Fax: +82-2-64902664; E-mail: kyryu@uos.ac.kr

http://dx.doi.org/10.5483/BMBRep.2014.47.9.128

Received 12 June 2014

Keywords: Dynamics, Homeostasis, Free ubiquitin, Polyubiquitin gene, Ubiquitin, Ubiquitin conjugates tion of the substrate by the $26 \mathrm{~S}$ proteasome, DNA repair, and activation of signal transduction pathways (3). Ub is removed from its target substrates by deubiquitinating enzymes (DUBs) after its functions are completed, and it is recycled back to the free $\mathrm{Ub}$ pool $(4,5)$. The free $\mathrm{Ub}$ pool is also maintained through the expression of two different classes of ubiquitin genes in mammals: monomeric Ub-ribosomal fusion genes, Uba52 and Uba80 (also known as Rps27a); and stress-inducible polyubiquitin genes, $U b b$ and $U b c$, composed of 3-4 and 9-10 tandem Ub coding units, respectively (6-8).

Ub inside cells is in dynamic equilibrium between "free" Ub that is readily available for ubiquitination and "conjugated" Ub that is covalently attached to target substrates. Recently, there has been a growing interest into how the free Ub pool is maintained under normal or stress conditions and how disruption of Ub pool dynamics and homeostasis affect the function and viability of cells. In this review, we briefly discuss recent advances in the field of Ub biology, focusing particularly on Ub homoeostasis, dynamic properties of cellular Ub pools, and the consequence of Ub deficiency or depletion in cells and metazoans.

\section{UBIQUITIN HOMEOSTASIS}

Ub plays diverse functions inside cells, and it is an abundant protein that comprises up to $5 \%$ of total proteins $(9,10)$. The substrate proteins of $\mathrm{Ub}$ are also abundant; therefore, the maintenance of cellular levels of $\mathrm{Ub}$, or Ub homeostasis, is important for timely ubiquitination of substrate proteins. One way to achieve Ub homeostasis is to regulate monomeric Ub-ribosomal fusion gene and polyubiquitin gene expression (Fig. 1).

Polyubiquitin genes are known to be upregulated under stress conditions, such as heat shock due to the presence of heat-shock response elements in promoter regions $(11,12)$. Another way to achieve $\mathrm{Ub}$ homeostasis is through regulation of DUBs that convert Ub conjugates into their monomeric free forms and increase levels of the free Ub pool (Fig. 1). There are nearly 100 DUBs in humans (13), and among these, $\mathrm{POH} 1$ (or Rpn11 in yeast) - the proteasome lid subunit in the 195 regulatory particle with DUB activity that detaches the polyubiquitin chain en bloc from target substrates during proteasomal degradation $(14,15)$ - generates unanchored free Ub chains.

In yeast, Doa4 was shown to be a DUB necessary for recycling $\mathrm{Ub}$ from polyubiquitinated proteins targeted to the proteasome for degradation (16). Doa4 was also shown to cleave

ISSN: 1976-670X (electronic edition)

Copyright (c) 2014 by the The Korean Society for Biochemistry and Molecular Biology

(ㄷ) This is an open-access article distributed under the terms of the Creative Commons Attribution Non-Commercial License (http://creativecommons.org/licenses/by-nc/3.0) which permits unrestricted non-commercial use, distribution, and reproduction in any medium, provided the original work is properly cited. 


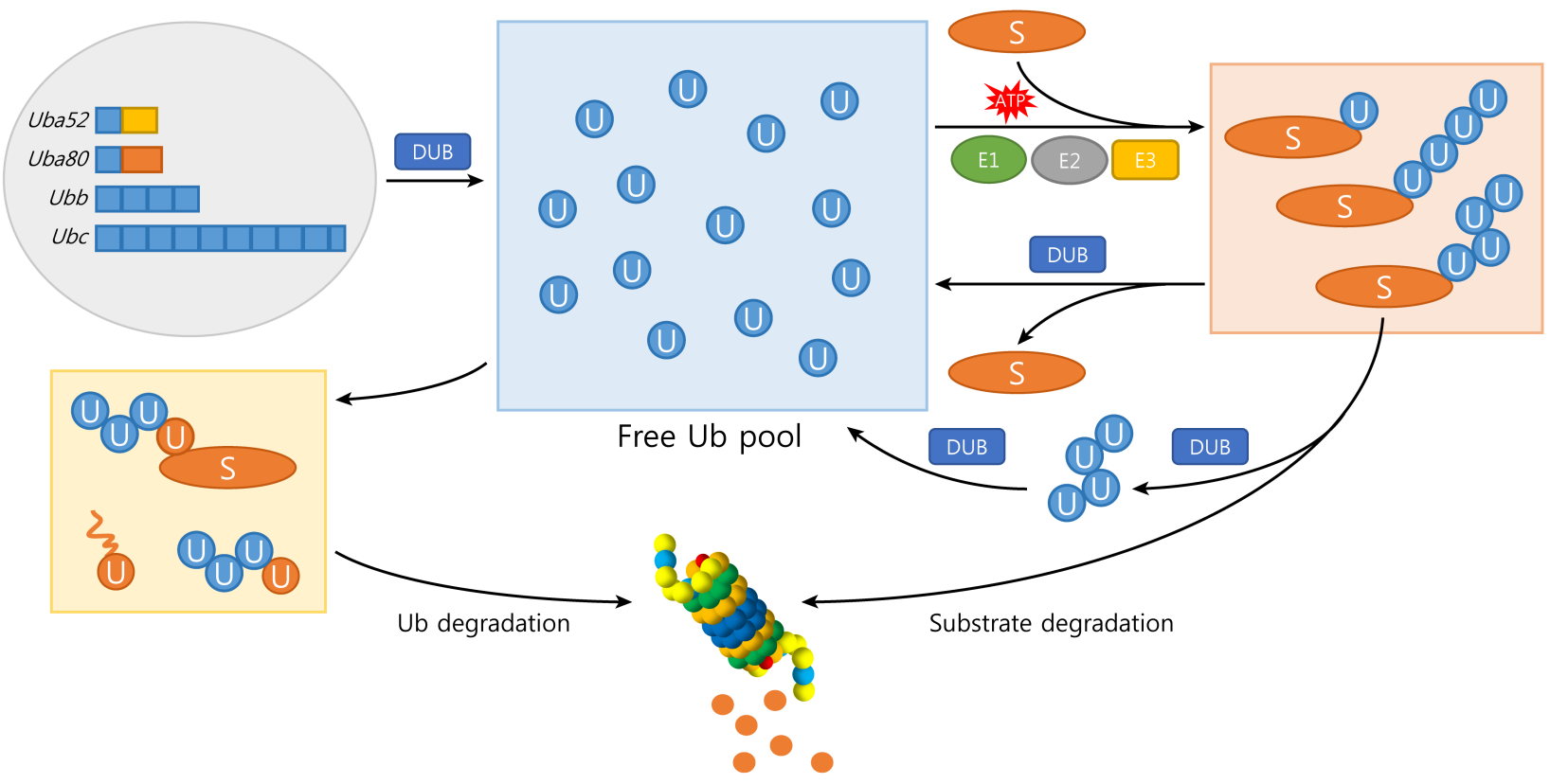

Fig. 1. Dynamic properties of cellular Ub pools. Conjugation of protein substrates (S) with Ub by a series of enzymes (E1-E3) and deconjugation by DUBs are in dynamic equilibrium. Ub conjugation results in monoubiquitination or polyubiquitination with different types of chain linkages. Ub conjugates or polyubiquitinated protein substrates with specific chain linkages (e.g., K48-linked chains) are targeted to the $26 \mathrm{~S}$ proteasome for degradation. During degradation of substrates, Ub chains are removed en bloc by a specific DUB (e.g., POH1) and are further processed to free $\mathrm{Ub}$. The free $\mathrm{Ub}$ pool is maintained by de novo Ub synthesis via expression of four different ubiquitin genes and by recycling or removal of Ub from Ub conjugates via actions of various DUBs (e.g., UCH37, USP14). Under certain conditions, Ub itself can also be degraded by the proteasome. See the text for details.

unanchored free Ub chains to generate monomeric free $\mathrm{Ub}$ (17). The loss of Doa4 or overexpression of its inhibitor Rfu1 (regulator of free ubiquitin chains 1) resulted in reduced levels of monomeric free $\mathrm{Ub}$ and disrupted Ub homeostasis, which rendered cells more susceptible to stress conditions.

The levels of free Ub can also be regulated by degradation of Ub itself (Fig. 1). In fact, Ub is a quite stable protein with a compact and globular structure resulting from significant intramolecular hydrogen bonding, with a short C-terminal tail that may serve as an inefficient target for the proteasome (18). However, Ub can also be degraded under certain conditions (19). First, although it may be a slow process, Ub itself can be polyubiquitinated by K48-linked chains and targeted to the proteasome. Second, Ub with a C-terminal tail longer than 20 amino acids with fused or conjugated peptides can be efficiently degraded by the proteasome in a polyubiquitination-independent manner. Third and most importantly, Ub, especially in the basal or proximal part of the polyubiquitin chain, can be degraded with its conjugated substrates.

The degradation of Ub itself is closely related to the activities of DUBs associated with the proteasome since the loss of USP14 (or Ubp6 in yeast) or UCH37 has been shown to trigger degradation of $\mathrm{Ub}$ along with its target substrates, leading to depletion of the free Ub pool (20-22). Intriguingly, Ub defi- ciency induced the upregulation of USP14 in mammals (Ryu KY, unpublished data) and Ubp6 in yeast (23), thereby sparing $\mathrm{Ub}$ from proteasomal degradation and increasing levels of free $\mathrm{Ub}$ in an attempt to reach Ub homeostasis.

\section{UBIQUITIN POOL DYNAMICS}

Cellular Ub pools are composed of Ub conjugated to target substrates via actions of enzymes E1-E3 and of unanchored free Ub synthesized de novo or recycled by DUBs. The dynamic properties of cellular Ub pools were first confirmed by microinjection of radiolabeled Ub into HeLa cells. Upon heat shock, levels of free Ub and ubiquitinated histones decreased and levels of high MW Ub conjugates increased (24). The increase of high MW Ub conjugates did not require synthesis of Ub de novo, but was rather due to redistribution of the existing free Ub pool. In fact, upon removal of heat shock, the levels of free Ub, ubiquitinated histones, and high MW Ub conjugates returned to those of normal conditions. Furthermore, when proteotoxic stress was induced by the presence of a proteasome inhibitor in cell lines stably expressing GFP-tagged $\mathrm{Ub}$, nuclear $\mathrm{Ub}$ conjugated to histone $2 \mathrm{~A}(\mathrm{uH} 2 \mathrm{~A})$ was redistributed to cytoplasmic Ub conjugates, demonstrating the dynamic nature of Ub pools (25). 
Various strategies have been employed to monitor the status of $\mathrm{Ub}$ in cells since cellular $\mathrm{Ub}$ pools are known to be dynamic rather than static. Immunoblot analysis via anti-Ub antibodies is a traditional method that detects free $\mathrm{Ub}$ and $\mathrm{Ub}$ conjugates. However, due to the nature of antibodies that prefer, but do not bind exclusively to, specific forms of Ub, an accurate determination of total Ub levels or of the ratio between free $\mathrm{Ub}$ and $\mathrm{Ub}$ conjugates has not been easy to achieve. To overcome this problem, a simple and reliable method was developed that quantifies the total Ub levels using indirect competitive ELISA (Ub-ELISA) after converting all Ub conjugates to monomeric free $\mathrm{Ub}$ using the Ub-specific protease Usp2-cc (10). However, Ub-ELISA has a limitation in that only the levels of total Ub can be determined accurately, whereas the mass spectrometry-based approach enables $\mathrm{Ub}$ researchers to quantify diverse cellular Ub species, as described below.

\section{PROTEOMIC APPROACH TO STUDY UBIQUITIN POOL DYNAMICS}

A proteomic approach was implemented to study protein ubiquitination at large scale. Trypsin digestion of ubiquitinated proteins or ubiquitinome yielded a signature peptide containing

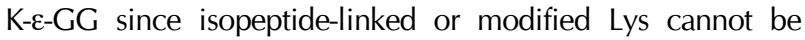
cleaved by trypsin (26). This Gly-Gly modification on Lys of the fragmented peptide can be detected via tandem mass spectrometry (MS/MS) due to a mass shift of $114 \mathrm{Da}$. Using this technique, more than 1,000 ubiquitinated proteins were identified in cell lysates from yeast expressing His-tagged Ub. Furthermore, 110 ubiquitination sites from 72 different proteins were identified (26). In particular, Ub itself was found to harbor 7 ubiquitination sites, suggesting a diverse nature of polyubiquitin chain assembly.

Since then, absolute quantification (AQUA), another proteomic strategy, has been developed to quantify the amount of
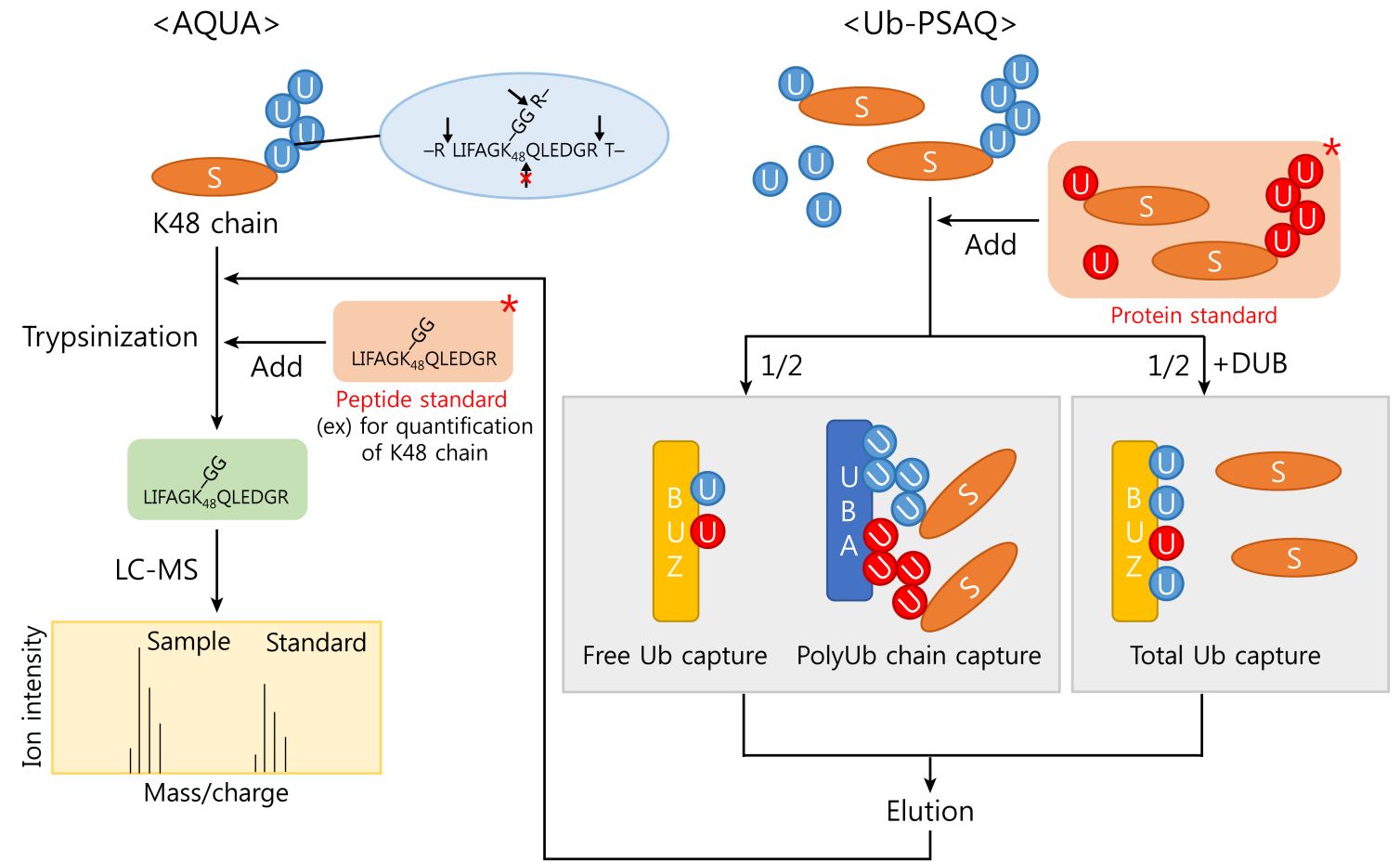

Fig. 2. Mass spectrometry-based approach to quantify cellular Ub pools. (Left) The amount of protein substrates (S) with specific Ub chains can be determined accurately via AQUA. After enriching ubiquitinated substrates, branched signature peptide fragments are generated by trypsin digestion since trypsin cannot cleave modified Lys. A known amount of stable isotope-labeled synthetic peptide standard (AQUA standard) is added into samples during trypsin digestion. Then, by comparing ion intensities, the signature peptide fragments are quantified after LC-MS analysis. For simplicity, only the K48-linked chains are shown. (Right) The amount of free Ub, total Ub, and protein substrates (S) with Ub chains can be quantified via Ub-PSAQ. Samples are mixed with a known amount of stable isotope-labeled protein recovery standard. Half of the sample is affinity-captured with a BUZ domain that specifically binds free Ub, followed by a UBA domain that specifically binds polyUb chains. The other half of the sample is deubiquitinated by treatment with Usp2-cc and is affinity-captured with the BUZ domain to quantify the total amount of Ub. After elution, the AQUA standard is added during trypsin digestion, and LC-MS analysis is carried out. Ion intensities of endogenous peptide fragments are compared to both the AQUA standard (for quantification of peptide fragments in the eluate) and protein standard (for determination of recovery from the affinity column), and the peptide fragments are quantified. 
polyubiquitin chains in biological specimens using liquid chromatography-tandem mass spectrometry (LC-MS/MS) $(27,28)$. In this strategy, ubiquitinated proteins are enriched, and then a known amount of stable isotope-labeled synthetic peptide standard is added, and the amount of branched peptide fragments generated by trypsin digestion is compared to that of the peptide standard. Through this method, the exact amount of specific polyubiquitin chains can be determined (Fig. 2). This strategy also facilitated the investigation of the dynamic nature of Ub pools in neurodegenerative diseases (29). Recently, antibodies that recognize the K-ع-GG peptide have been developed (30), facilitating pull-down and enriching K-ع-GG peptides from ubiquitinated proteins in cell lysates, and the dynamic changes in $\mathrm{Ub}$ pools and ubiquitination of proteins were observed by enriching K-ع-GG peptides in stable isotope labeling with amino acids in cell culture (SILAC)-encoded cells under proteasome or DUB inhibition (31).

\section{DISTRIBUTION OF CELLULAR UBIQUITIN POOLS}

In addition to the quantification of polyubiquitin chains, protein standard absolute quantification (Ub-PSAQ) - a method for accurate measurement of diverse cellular Ub pools - was also developed (32). In this method, free Ub species are affinity-captured with Ub-binding $\mathrm{Zn}$ finger (BUZ) domain, and then polyubiquitin chains are affinity-captured with Ub-associated (UBA) domain (Fig. 2). To determine the amount of total $\mathrm{Ub}, \mathrm{Usp} 2-\mathrm{cc}$ is treated to convert all forms of Ub conjugates to monomeric free $\mathrm{Ub}$, and free $\mathrm{Ub}$ is then captured with the BUZ domain. After elution and trypsin digestion, peptide fragments are analyzed via liquid chromatography-electrospray ionization time-of-flight mass spectrometry (LC-ESI TOF MS) and are quantified by comparing to stable isotope-labeled peptides recovered from free $\mathrm{Ub}$ and $\mathrm{Ub}$ conjugate standards that are spiked into the samples before affinity capture. The concentration of monoubiquitinated species is determined by subtracting the amount of free $\mathrm{Ub}$ and polyubiquitin chains from that of the total Ub.

According to the results of Ub-PSAQ, more than $20 \%$ of total Ub pools were composed of free Ub, and more than $60 \%$ were composed of monoubiquitinated proteins in HEK293 cells and mouse embryonic fibroblasts (MEFs), suggesting that proteins with the polyubiquitin chain are less than $20 \%$ of the total Ub pools. Surprisingly, Ub pool distribution was different for brain tissues when compared to HEK293 cells and MEFs. In mouse brains, $60 \%$ of the total $\mathrm{Ub}$ pools were free $\mathrm{Ub}$ and proteins with polyubiquitin chains were only $5 \%$ of the composition. In the human frontal cortex, free Ub content was even higher, up to $80 \%$ (32). The immunoblot analysis was consistent with the results of Ub-PSAQ data and also revealed



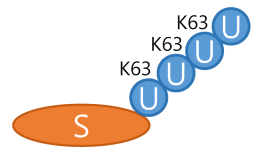

K63 chain

NF-KB activation

DNA repair

Lysosomal targeting

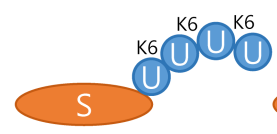

K6 chain

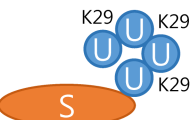

K29 chain

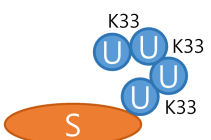

K33 chain

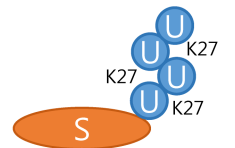

K27 chain

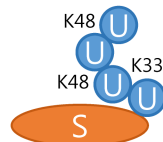

Heterogeneous chain

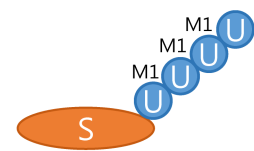

Linear chain

NF-KB activation



Branched/Forked chain

Unknown functions

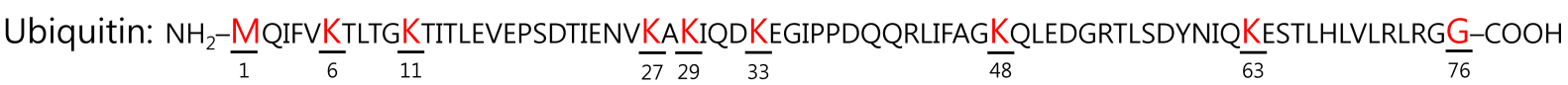

Fig. 3. Various types of ubiquitination and the specific role of Ub chain linkages. Ub is composed of 76 amino acids and forms isopeptide linkages between the carboxyl group of Gly76 residue and $\varepsilon$-amino group of Lys residue in substrate or in another Ub molecule. The substrate can be monoubiquitinated, multi-monoubiquitinated, or polyubiquitinated with different types of chain linkages. The $1^{\text {st }}$ Met and seven Lys residues in Ub (Lys6, Lys11, Lys27, Lys29, Lys33, Lys48, and Lys63) can all form specific chain linkages with distinct conformations and can exert specific (or unknown) functions. Although it is rare, heterogeneous and branched/forked chain linkages are also possible. 
that levels of free $\mathrm{Ub}$ in mouse brain and cultured neurons were much higher than those in HEK293 cells and MEFs (Ryu $\mathrm{KY}$, unpublished data). Therefore, cellular contents of different Ub species may vary depending on the type of cells, and high levels of free $\mathrm{Ub}$ in neurons suggest an important role of $\mathrm{Ub}$ for neuronal function and survival.

\section{COMPLEXITY AND SPECIFIC ROLE OF UBIQUITIN CHAIN LINKAGES}

Although polyubiquitinated proteins may not be abundant in any cell types under normal conditions, they constitute a significant portion of total $\mathrm{Ub}$ pools under stress conditions, and the effect of polyubiquitination on target proteins are different depending on the type of $\mathrm{Ub}$ chain linkages. To investigate the role of Ub chain linkages, polyubiquitin chain-specific antibodies were developed which recognize specific types of chains such as K48- or K63-linked (33). These facilitated research investigating mechanisms of polyubiquitin chain assembly (Fig. 3).

However, the dynamic nature of chain elongation and shortening, the formation of branched or forked Ub chains, and even the formation of heterogeneous chains that contain a mixture of different types of linkages have hampered Ub researchers from obtaining a complete understanding of the link between chain formations and their biological consequences (34-36). Nevertheless, K48- or K63-linked polyubiquitin chains are well known for their respective roles in proteasomal degradation or in other non-proteasomal cellular processes.

The roles of other unconventional polyubiquitin chains (K6, $\mathrm{K} 11, \mathrm{~K} 27, \mathrm{~K} 29$, or K33) have also been investigated. For example, K48-linked polyubiquitin chains as well as an accumulation of K11-linked polyubiquitin chains were observed in yeast upon inhibition of the proteasome, and Ubc6 was identified via quantitative proteomic analysis using LC-MS/MS as a target substrate for polyubiquitination with K11 linkages. Interestingly, Ubc6 also served as an E2 enzyme in conjunction with an E3 ligase Doa10 to form K11-linked polyubiquitin chains on misfolded ER proteins and for targeting them to the proteasome for degradation. This is called the endoplasmic reticulum-associated degradation (ERAD) pathway and K11R mutant, which cannot form $\mathrm{K} 11$ linkages, were shown to be more sensitive to ER stress (37). A quadruple mutation (K6R, K11R, $\mathrm{K} 27 \mathrm{R}$, and K63R) in yeast increased the levels of K33-linked polyubiquitin chains by more than 20-fold, suggesting the redundant role of different chain linkages. However, this was not sufficient to fully protect cells from reduced proliferation and viability in quadruple mutants (37). Therefore, although all non-K63 linkages may serve as a signal to target proteins for proteasomal degradation, specific target proteins may need to be polyubiquitinated with different chain linkages.

\section{ALTERATIONS IN UBIQUITIN POOLS VIA DELETION OF POLYUBIQUITIN GENES}

Alterations in Ub pools and on its phenotypic consequences were investigated through the deletion of polyubiquitin genes in yeast and in mammals. The deletion of the stress-inducible polyubiquitin gene UBI4 did not affect the viability of yeast under normal vegetative growth conditions but did cause the yeast to exhibit an increased sensitivity against heat shock, starvation, and other stress conditions (38).

In addition, the depletion of free Ub via cycloheximide treatment caused toxicity with reduced viability in yeast (39). Mutations in the proteasome that spare $\mathrm{Ub}$ from degradation or overexpression of $\mathrm{Ub}$ in yeast conferred resistance against cycloheximide-induced toxicity. Although it was expected for the steady-state levels of many proteins to be affected by the inhibition of de novo protein synthesis by cycloheximide treatment, it is quite surprising that perturbations in Ub homeostasis are most critical for the viability of yeast. These results indicate that maintaining adequate levels of free $\mathrm{Ub}$, which could be rate limiting, is essential for cellular survival.

In mice, there are two polyubiquitin genes, Ubb and Ubc, and they were shown to be required even under normal growth conditions. Homozygous deletion of Ubc caused mid-gestation embryonic lethality due to defective proliferation of hepatocytes and bipotent fetal liver epithelial progenitor cells $(40,41)$. This result was surprising because, based on previous studies on yeast, it had been believed that the polyubiquitin gene is required under stress conditions, but is dispensable under normal growth conditions (38). On the other hand, homozygous deletion of Ubb caused infertility and adult-onset hypothalamic neurodegeneration with metabolic and sleep abnormalities (42-44). These phenotypes in Ubb knockout mice are closely associated with the expression profiles of $U b b$ since $U b b$ is highly expressed in germ cells and in hypothalamic neurons. Therefore, the disruption of Ubb reduced the levels of Ub most dramatically for these specific cell types. Intriguingly, Ubb was also highly expressed in the locus coeruleus region of the brain. However, locus coeruleus neurons were well-protected, even when Ubb was disrupted (45). Although total Ub levels were reduced by $30 \%$ in both the hypothalamus and in the locus coeruleus of Ubb knockout mice, free Ub levels were reduced by $40 \%$ only in the hypothalamus of Ubb knockout mice but not in the locus coeruleus. These observations suggest that the levels of free $\mathrm{Ub}$ in neurons or in neighboring cells are important for neuronal function and survival.

In Ubc knockout MEFs, the total Ub levels were reduced by $40 \%$, reflecting the significance of Ubc contribution toward total Ub levels in MEFs. The deletion of Ubc in MEFs caused reduced proliferation, premature senescence, and delayed entry into mitosis (40). Therefore, the relative contributions of $U b b$ and Ubc toward the total Ub levels, resulting from their transcriptional activities, are different depending on the types of 
cells or tissues. Due to different tissue spectrum of Ubb and Ubc expression, the deletion of one polyubiquitin gene may not necessarily be fully compensated for by the upregulation of another polyubiquitin gene. As cell cycle progression can be regulated by the expression levels of polyubiquitin genes and increased levels of Ub are closely associated with the proliferation of cancer cells, knockdown of Ubb has been shown to induce apoptosis in cancer cells (46). Interestingly, knockdown of Ubb dramatically reduced the levels of free Ub, but not of Ub conjugates. Therefore, levels of free $\mathrm{Ub}$ are closely associated with cell viability.

\section{ALTERATIONS IN UBIQUITIN POOLS VIA MUTATIONS IN DEUBIQUITINATING ENZYMES}

Ub pools can also be regulated by DUBs. DUBs are classified into five families based on their domains, and these are known as Ub C-terminal hydrolase (UCH), Ub-specific protease (USP), ovarian tumor (OTU), Josephin (MJD), and JAB1/MPN/Mov34 metalloenzyme (JAMM) (13). Among these, Ub C-terminal hydrolase L1 (UCH-L1) is one of the most abundant proteins in the brain, comprising up to $5 \%$ of the total of soluble proteins (47). Mutations in Uchl1 are closely linked to the susceptibility of humans to Parkinson's disease (PD) (48). In mice, deletion or loss-of-function mutations in Uch/1 caused gracile axonal dystrophy (gad), which is an autosomal recessive disorder with sensory and motor ataxia (49).

Due to dysfunction of UCH-L1 in gad mice, the free Ub pool was disturbed, leading to an accumulation of Ub-positive abnormal protein deposits in the nervous system. The importance of UCH-L1 has also been implicated in the pathogenesis of Alzheimer's disease (AD) (50). Soluble UCH-L1 protein levels, but not mRNA levels, were reduced in AD model APP/PS1 mice, and the exogenous expression of UCH-L1 improved synaptic function and contextual learning in APP/PS1 mice. In gad mice, the levels of free $\mathrm{Ub}$ in the brain were significantly reduced, but levels of Ub conjugates or ubiquitin gene expression were not affected (51). Therefore, UCH-L1 exerts an essential role in maintaining free Ub levels.

Mutations in Usp14, which encodes Ub-specific protease, are also known to cause neurological disorders, including synaptic dysfunction (52). Since the ataxia (ax) gene encodes USP14, the expression of intact USP14 protein was not observed in $a x^{\prime}$ mice. Free $U b$, but not $U b$ conjugate, levels were reduced in the brain of $a x^{\prime}$ mice, but neuron-specific expression of the Usp14 transgene was sufficient to restore free Ub levels in the spinal cord and motor neuron axons and could rescue ataxia phenotypes with defective neuromuscular junctions in $a x^{\prime}$ mice $(53,54)$. In $a x^{\prime}$ mice, the levels of free Ub were reduced by $30 \%$, not only in the brain, but also in other tissues such as the liver, heart, and spleen (20). In synaptosomal fractions of $a x^{\prime}$ mice, however, the levels of free Ub were further reduced by $60 \%$, with a concomitant reduction in Ub conjugates by $40 \%$, suggesting there to be a significant impair- ment in Ub homeostasis at the synapse (53). Since free Ub needs to be transported along the axon down to the synapse, and this axonal transport of free $\mathrm{Ub}$ is a quite slow process (55), the levels of synaptic Ub could be further reduced due to the dysfunction of USP14. Synaptic dysfunction in $a x^{\prime}$ mice was also rescued by neuron-specific expression of ubiquitin transgene, which restored levels of free $\mathrm{Ub}$ (56).

Although mutations in two different deubiquitinating enzymes UCH-L1 and USP14 lead to neurological abnormalities in mice with reduced levels of free $\mathrm{Ub}$, their effects on the viability of mice and on the onset of symptoms were much more severe, and the altered synaptic transmission in the hippocampus was only observed when Usp14 was mutated or lost (57). Since loss-of-function mutations in Usp14 upregulate the expression of proteasomal subunits, and mutations in Uch/1 upregulate the expression of lysosomal proteases, each DUB may be involved in the regulation of Ub pools in different subcellular compartments.

\section{CONCLUDING REMARKS}

As briefly summarized in this review, numerous research has been carried out during the past three decades to investigate cellular Ub pools, revealing the importance of polyubiquitin genes and of DUBs in maintaining threshold levels of the free Ub pool. Since cells may not distinguish polyubiquitin gene-encoded $\mathrm{Ub}$ from monoubiquitin gene-encoded $\mathrm{Ub}$ after the deubiquitination of $\mathrm{Ub}$ adduct or Ub-ribosomal fusion proteins, the outcome of polyubiquitin gene deletion is very simple in that the availability of cellular $\mathrm{Ub}$ is reduced. It is interesting to note that the reduced availability of $U b$ is sufficient to cause adverse effects on cellular function and survival. In particular, a reduced viability of cells due to disruption of Ub homeostasis accompanied a concomitant reduction of free Ub levels. Although it has yet to be determined whether or not disruption of $\mathrm{Ub}$ homeostasis is the cause or the effect of neuropathological abnormalities observed in polyubiquitin gene-deficient models, it is quite reasonable to speculate that levels of free $\mathrm{Ub}$ are an important determinant of cellular vulnerability. Furthermore, as a result of the dynamic properties of Ub pools, free $\mathrm{Ub}$ can be converted to Ub conjugates, which sometimes form polyubiquitinated protein deposits under stress conditions. Abnormal protein deposits tend to be clustered with reduced solubility. Therefore, sequestration of free $\mathrm{Ub}$ into insoluble fractions can also reduce the availability of free $\mathrm{Ub}$ and can disrupt Ub homeostasis, potentially affecting various cellular processes that require protein ubiquitination or degradation. The development of a strategy to maintain Ub homeostasis (above the threshold levels) under stress conditions is required to protect cells from various extracellular insults.

\section{ACKNOWLEDGEMENTS}

This work was supported by the 2012 Research Fund of the 
University of Seoul to K. Y. R.

\section{REFERENCES}

1. Hershko, A. and Ciechanover, A. (1998) The ubiquitin system. Annu. Rev. Biochem. 67, 425-479.

2. Hicke, L. (2001) Protein regulation by monoubiquitin. Nat. Rev. Mol. Cell Biol. 2, 195-201.

3. Pickart, C. M. and Fushman, D. (2004) Polyubiquitin chains: polymeric protein signals. Curr. Opin. Chem. Biol. 8, 610-616.

4. Amerik, A. Y. and Hochstrasser, M. (2004) Mechanism and function of deubiquitinating enzymes. Biochim. Biophys. Acta 1695, 189-207.

5. Komander, D., Clague, M. J. and Urbe, S. (2009) Breaking the chains: structure and function of the deubiquitinases. Nat. Rev. Mol. Cell Biol. 10, 550-563.

6. Wiborg, O., Pedersen, M. S., Wind, A., Berglund, L. E., Marcker, K. A. and Vuust, J. (1985) The human ubiquitin multigene family: some genes contain multiple directly repeated ubiquitin coding sequences. EMBO J. 4, 755-759.

7. Baker, R. T. and Board, P. G. (1991) The human ubiquitin-52 amino acid fusion protein gene shares several structural features with mammalian ribosomal protein genes. Nucleic Acids Res. 19, 1035-1040.

8. Redman, K. L. and Rechsteiner, M. (1989) Identification of the long ubiquitin extension as ribosomal protein S27a. Nature 338, 438-440.

9. Ohtani-Kaneko, R., Asahara, M., Takada, K., Kanda, T., ligo, M., Hara, M., Yokosawa, H., Ohkawa, K. and Hirata, K. (1996) Nerve growth factor (NGF) induces increase in multi-ubiquitin chains and concomitant decrease in free ubiquitin in nuclei of PC12h. Neurosci. Res. 26, 349-355.

10. Ryu, K. Y., Baker, R. T. and Kopito, R. R. (2006) Ubiquitin-specific protease 2 as a tool for quantification of total ubiquitin levels in biological specimens. Anal. Biochem. 353, 153-155.

11. Fornace, A. J., Jr., Alamo, I., Jr., Hollander, M. C. and Lamoreaux, E. (1989) Ubbiquitin mRNA is a major stress-induced transcript in mammalian cells. Nucleic Acids Res. 17, 1215-1230.

12. Bond, U. and Schlesinger, M. J. (1986) The chicken ubiquitin gene contains a heat shock promoter and expresses an unstable mRNA in heat-shocked cells. Mol. Cell. Biol. 6, 4602-4610.

13. Reyes-Turcu, F. E., Ventii, K. H. and Wilkinson, K. D. (2009) Regulation and cellular roles of ubiquitin-specific deubiquitinating enzymes. Annu. Rev. Biochem. 78, 363-397.

14. Yao, T. and Cohen, R. E. (2002) A cryptic protease couples deubiquitination and degradation by the proteasome. Nature 419, 403-407.

15. Verma, R., Aravind, L., Oania, R., McDonald, W. H., Yates, J. R., 3rd, Koonin, E. V. and Deshaies, R. J. (2002) Role of Rpn11 metalloprotease in deubiquitination and degradation by the $26 \mathrm{~S}$ proteasome. Science 298, 611-615.

16. Swaminathan, S., Amerik, A. Y. and Hochstrasser, M. (1999) The Doa4 deubiquitinating enzyme is required for ubiquitin homeostasis in yeast. Mol. Biol. Cell 10, 2583-2594.
17. Kimura, Y., Yashiroda, H., Kudo, T., Koitabashi, S., Murata, S., Kakizuka, A. and Tanaka, K. (2009) An inhibitor of a deubiquitinating enzyme regulates ubiquitin homeostasis. Cell 137, 549-559.

18. Vijay-Kumar, S., Bugg, C. E. and Cook, W. J. (1987) Structure of ubiquitin refined at $1.8 \mathrm{~A}$ resolution. J. Mol. Biol. 194, 531-544.

19. Weissman, A. M., Shabek, N. and Ciechanover, A. (2011) The predator becomes the prey: regulating the ubiquitin system by ubiquitylation and degradation. Nat. Rev. Mol. Cell Biol. 12, 605-620.

20. Anderson, C., Crimmins, S., Wilson, J. A., Korbel, G. A., Ploegh, H. L. and Wilson, S. M. (2005) Loss of Usp14 results in reduced levels of ubiquitin in ataxia mice. J. Neurochem. 95, 724-731.

21. Leggett, D. S., Hanna, J., Borodovsky, A., Crosas, B., Schmidt, M., Baker, R. T., Walz, T., Ploegh, H. and Finley, D. (2002) Multiple associated proteins regulate proteasome structure and function. Mol. Cell 10, 495-507.

22. Lee, M. J., Lee, B. H., Hanna, J., King, R. W. and Finley, D. (2011) Trimming of ubiquitin chains by proteasome-associated deubiquitinating enzymes. Mol. Cell. Proteomics 10, R110 003871.

23. Hanna, J., Meides, A., Zhang, D. P. and Finley, D. (2007) A ubiquitin stress response induces altered proteasome composition. Cell 129, 747-759.

24. Carlson, N., Rogers, S. and Rechsteiner, M. (1987) Microinjection of ubiquitin: changes in protein degradation in HeLa cells subjected to heat-shock. J. Cell Biol. 104, 547-555.

25. Dantuma, N. P., Groothuis, T. A., Salomons, F. A. and Neefjes, J. (2006) A dynamic ubiquitin equilibrium couples proteasomal activity to chromatin remodeling. J. Cell Biol. 173, 19-26.

26. Peng, J., Schwartz, D., Elias, J. E., Thoreen, C. C., Cheng, D., Marsischky, G., Roelofs, J., Finley, D. and Gygi, S. P. (2003) A proteomics approach to understanding protein ubiquitination. Nat. Biotechnol. 21, 921-926.

27. Kirkpatrick, D. S., Denison, C. and Gygi, S. P. (2005) Weighing in on ubiquitin: the expanding role of mass-spectrometry-based proteomics. Nat. Cell Biol. 7, 750-757.

28. Kirkpatrick, D. S., Gerber, S. A. and Gygi, S. P. (2005) The absolute quantification strategy: a general procedure for the quantification of proteins and post-translational modifications. Methods 35, 265-273.

29. Bennett, E. J., Shaler, T. A., Woodman, B., Ryu, K. Y., Zaitseva, T. S., Becker, C. H., Bates, G. P., Schulman, H. and Kopito, R. R. (2007) Global changes to the ubiquitin system in Huntington's disease. Nature 448, 704-708.

30. Kim, W., Bennett, E. J., Huttlin, E. L., Guo, A., Li, J., Possemato, A., Sowa, M. E., Rad, R., Rush, J., Comb, M. J., Harper, J. W. and Gygi, S. P. (2011) Systematic and quantitative assessment of the ubiquitin-modified proteome. Mol. Cell 44, 325-340.

31. Udeshi, N. D., Mani, D. R., Eisenhaure, T., Mertins, P., Jaffe, J. D., Clauser, K. R., Hacohen, N. and Carr, S. A. (2012) Methods for quantification of in vivo changes in protein ubiquitination following proteasome and deubiquitinase inhibition. Mol. Cell. Proteomics 11, 148-159. 
32. Kaiser, S. E., Riley, B. E., Shaler, T. A., Trevino, R. S., Becker, C. H., Schulman, H. and Kopito, R. R. (2011) Protein standard absolute quantification (PSAQ) method for the measurement of cellular ubiquitin pools. Nat. Methods 8, 691-696.

33. Newton, K., Matsumoto, M. L., Wertz, I. E., Kirkpatrick, D. S., Lill, J. R., Tan, J., Dugger, D., Gordon, N., Sidhu, S. S., Fellouse, F. A., Komuves, L., French, D. M., Ferrando, R. E., Lam, C., Compaan, D., Yu, C., Bosanac, I., Hymowitz, S. G., Kelley, R. F. and Dixit, V. M. (2008) Ubiquitin chain editing revealed by polyubiquitin linkage-specific antibodies. Cell 134, 668-678.

34. Komander, D. (2009) The emerging complexity of protein ubiquitination. Biochem. Soc. Trans. 37, 937-953.

35. Ye, Y. and Rape, M. (2009) Building ubiquitin chains: E2 enzymes at work. Nat. Rev. Mol. Cell Biol. 10, 755-764.

36. Husnjak, K. and Dikic, I. (2012) Ubiquitin-binding proteins: decoders of ubiquitin-mediated cellular functions. Annu. Rev. Biochem. 81, 291-322.

37. Xu, P., Duong, D. M., Seyfried, N. T., Cheng, D., Xie, Y., Robert, J., Rush, J., Hochstrasser, M., Finley, D. and Peng, J. (2009) Quantitative proteomics reveals the function of unconventional ubiquitin chains in proteasomal degradation. Cell 137, 133-145.

38. Finley, D., Ozkaynak, E. and Varshavsky, A. (1987) The yeast polyubiquitin gene is essential for resistance to high temperatures, starvation, and other stresses. Cell 48, 1035-1046.

39. Hanna, J., Leggett, D. S. and Finley, D. (2003) Ubiquitin depletion as a key mediator of toxicity by translational inhibitors. Mol. Cell. Biol. 23, 9251-9261.

40. Ryu, K. Y., Maehr, R., Gilchrist, C. A., Long, M. A., Bouley, D. M., Mueller, B., Ploegh, H. L. and Kopito, R. R. (2007) The mouse polyubiquitin gene UbC is essential for fetal liver development, cell-cycle progression and stress tolerance. EMBO J. 26, 2693-2706.

41. Park, H., Yoon, M. S. and Ryu, K. Y. (2013) Disruption of polyubiquitin gene Ubc leads to defective proliferation of hepatocytes and bipotent fetal liver epithelial progenitor cells. Biochem. Biophys. Res. Commun. 435, 434-440.

42. Ryu, K. Y., Fujiki, N., Kazantzis, M., Garza, J. C., Bouley, D. M., Stahl, A., Lu, X. Y., Nishino, S. and Kopito, R. R. (2010) Loss of polyubiquitin gene Ubb leads to metabolic and sleep abnormalities in mice. Neuropathol. Appl. Neurobiol. 36, 285-299.

43. Ryu, K. Y., Garza, J. C., Lu, X. Y., Barsh, G. S. and Kopito, R. R. (2008) Hypothalamic neurodegeneration and adult-onset obesity in mice lacking the Ubb polyubiquitin gene. Proc. Natl. Acad. Sci. U.S.A. 105, 4016-4021.

44. Ryu, K. Y., Sinnar, S. A., Reinholdt, L. G., Vaccari, S., Hall, S., Garcia, M. A., Zaitseva, T. S., Bouley, D. M., Boekelheide, K., Handel, M. A., Conti, M. and Kopito, R. R. (2008) The mouse polyubiquitin gene Ubb is essential for meiotic progression. Mol. Cell. Biol. 28, 1136-1146.

45. Park, C. W., Ryu, H. W. and Ryu, K. Y. (2012) Locus coeruleus neurons are resistant to dysfunction and degeneration by maintaining free ubiquitin levels although total ubiquitin levels decrease upon disruption of polyubiquitin gene Ubb. Biochem. Biophys. Res. Commun. 418, 541-546.

46. Oh, C., Park, S., Lee, E. K. and Yoo, Y. J. (2013) Downre- gulation of ubiquitin level via knockdown of polyubiquitin gene Ubb as potential cancer therapeutic intervention. Sci. Rep. 3, 2623.

47. Wilkinson, K. D., Lee, K. M., Deshpande, S. Duerksen-Hughes, P., Boss, J. M. and Pohl, J. (1989) The neuron-specific protein PGP 9.5 is a ubiquitin carboxyl-terminal hydrolase. Science 246, 670-673.

48. Liu, Y., Fallon, L., Lashuel, H. A., Liu, Z. and Lansbury, P. T., Jr. (2002) The UCH-L1 gene encodes two opposing enzymatic activities that affect alpha-synuclein degradation and Parkinson's disease susceptibility. Cell 111, 209-218.

49. Saigoh, K., Wang, Y. L., Suh, J. G., Yamanishi, T., Sakai, Y., Kiyosawa, H., Harada, T., Ichihara, N., Wakana, S., Kikuchi, T. and Wada, K. (1999) Intragenic deletion in the gene encoding ubiquitin carboxy-terminal hydrolase in gad mice. Nat. Genet. 23, 47-51.

50. Gong, B., Cao, Z., Zheng, P., Vitolo, O. V., Liu, S., Staniszewski, A., Moolman, D., Zhang, H., Shelanski, M. and Arancio, O. (2006) Ubiquitin hydrolase Uch-L1 rescues beta-amyloid-induced decreases in synaptic function and contextual memory. Cell 126, 775-788.

51. Osaka, H., Wang, Y. L., Takada, K., Takizawa, S., Setsuie, R., Li, H., Sato, Y., Nishikawa, K., Sun, Y. J., Sakurai, M., Harada, T., Hara, Y., Kimura, I., Chiba, S., Namikawa, K., Kiyama, H., Noda, M., Aoki, S. and Wada, K. (2003) Ubiquitin carboxy-terminal hydrolase L1 binds to and stabilizes monoubiquitin in neuron. Hum. Mol. Genet. 12, 1945-1958.

52. Wilson, S. M., Bhattacharyya, B., Rachel, R. A., Coppola, V., Tessarollo, L., Householder, D. B., Fletcher, C. F. Miller, R. J., Copeland, N. G. and Jenkins, N. A. (2002) Synaptic defects in ataxia mice result from a mutation in Usp14, encoding a ubiquitin-specific protease. Nat. Genet. 32, 420-425.

53. Chen, P. C., Qin, L. N., Li, X. M., Walters, B. J., Wilson, J. A., Mei, L. and Wilson, S. M. (2009) The proteasome-associated deubiquitinating enzyme Usp14 is essential for the maintenance of synaptic ubiquitin levels and the development of neuromuscular junctions. J. Neurosci. 29, 10909-10919.

54. Crimmins, S., Jin, Y., Wheeler, C., Huffman, A. K., Chapman, C., Dobrunz, L. E., Levey, A., Roth, K. A., Wilson, J. A. and Wilson, S. M. (2006) Transgenic rescue of ataxia mice with neuronal-specific expression of ubiquitin-specific protease 14. J. Neurosci. 26, 11423-11431.

55. Bizzi, A., Schaetzle, B., Patton, A., Gambetti, P. and Autilio-Gambetti, L. (1991) Axonal transport of two major components of the ubiquitin system: free ubiquitin and ubiquitin carboxyl-terminal hydrolase PGP 9.5. Brain Res. 548, 292-299.

56. Chen, P. C., Bhattacharyya, B. J., Hanna, J., Minkel, H., Wilson, J. A., Finley, D., Miller, R. J. and Wilson, S. M. (2011) Ubiquitin homeostasis is critical for synaptic development and function. J. Neurosci. 31, 17505-17513.

57. Walters, B. J., Campbell, S. L., Chen, P. C., Taylor, A. P., Schroeder, D. G., Dobrunz, L. E., Artavanis-Tsakonas, K., Ploegh, H. L., Wilson, J. A., Cox, G. A. and Wilson, S. M. (2008) Differential effects of Usp14 and Uch-L1 on the ubiquitin proteasome system and synaptic activity. Mol. Cell. Neurosci. 39, 539-548. 